\title{
Reversal mechanism and suppression of training in an exchange-coupled system
}

\author{
Amitesh Paul* \\ Institut für Festkörperforschung IFF-4 “Streumethoden,” Forschungszentrum Jülich GmbH, D-52425 Jülich, Germany \\ Claus M. Schneider \\ Institut für Festkörperforschung IFF-9 "Elektronische Eigenschaften" and CNI-Center for Nanoelectronic Systems in Information \\ Technology, Forschungszentrum Jülich GmbH, D-52425 Jülich, Germany \\ Jochen Stahn \\ Laboratory for Neutron Scattering, ETH Zurich \& PSI, Paul Scherrer Institut, CH-5232 Villigen PSI, Switzerland \\ (Received 25 April 2007; revised manuscript received 23 May 2007; published 20 November 2007)
}

\begin{abstract}
We show that the extent of training in exchange-biased systems can be strongly influenced by the field cooling procedure. This phenomenon is revealed by comparing the behavior of the system in two different magnetic configurations: cooling the system in a remanent state without an external magnetic field (state I) results in a suppression of the training effect, whereas the same sample being field cooled (state II) exhibits a clear training response. Interestingly, by cooling the sample in a critical field $H_{\mathrm{FC}}$ close to the coercive field $H_{\mathrm{C}}$ of the ferromagnet, we find a peculiar situation with a coexistence of states I and II. By using a depth-sensitive polarized neutron reflectivity technique, we can establish a clear correlation of the reversal mechanism with either the untrained or trained state.
\end{abstract}

DOI: 10.1103/PhysRevB.76.184424

PACS number(s): 75.70.Cn, 75.60.Jk

\section{INTRODUCTION}

Direct exchange coupling across the interface between a ferromagnet (FM) and an antiferromagnet (AF) gives rise to magnetic proximity effects. These usually affect the magnetic behavior of both the FM and AF, for example, with respect to the ordering temperatures, or the magnitude and orientation of the magnetic moments. In particular, the interfacial exchange coupling can "lock" the magnetization into the FM in a well-defined direction. This effect, which in a phenomenological picture takes the form of a unidirectional magnetic anisotropy is also known as exchange bias. ${ }^{1}$ The exchange biasing is usually established and controlled by heating the FM/AF system above and subsequently cooling it below the blocking temperature $T_{B}$ of the AF-generally in the presence of an external field $H_{\mathrm{FC}}$. By means of this fieldcooling procedure the spins at the FM/AF interface are "frozen" in a predefined direction. Exchange biasing has a significant technological importance as it is widely employed to define reference magnetization directions in spin-valve elements and magnetic tunnel junctions, which are key devices in magnetic sensorics and high-density magnetic data storage.

The basic mechanism of exchange bias with respect to its magnetization reversal (symmetric and asymmetric) is fairly well understood for model systems ${ }^{2}$ or in general. ${ }^{3}$ However, a comprehension of many other interesting aspects such as the distribution of uncompensated spins at the interface and the exchange interaction of the FM with the uncompensated spins $^{4,5}$ still remains elusive for half a century now. The phenomenon of exchange bias depends predominantly upon the state of the interface, at which the AF-FM spins are frozen in, as they are field cooled, provided that the AF domain size remains unchanged with respect to its initial state. An irreversible (pinned) AF interface magnetization arising from the volume domains stabilized by the defects in the AF has been a crucial ingredient in explaining the origin of the effect. ${ }^{6}$ The magnetization state of the FM can be modified by an external magnetic field $H_{\mathrm{FC}}$ applied during the cooling/ growth of the interface. Thus, the strength of $H_{\mathrm{FC}}$ determines the bias field $\left(H_{\mathrm{EB}}\right)$ values and can also influence the magnetization reversal process. $\mathrm{Co}(\mathrm{FM}) / \mathrm{CoO}(\mathrm{AF})$ bilayers are among the most widely studied systems, ${ }^{7-10}$ and exhibit the typically observed "negative exchange bias" (a shift of the hysteresis loop in the direction opposite to $H_{\mathrm{FC}}$ ). This is shown in Fig. 1(a), where a cooling field of $H_{\mathrm{FC}}=-4.0 \mathrm{kOe}$ was used and the hysteresis loops for the first and second field cycles have been measured. Thus, this result is a typical example of exchange bias being determined by the strength of the cooling field.

However, it has also been shown explicitly, ${ }^{11,12}$ that it is the magnetization state of the FM caused by the external field rather than the cooling field itself, that defines the interface coupling in AF-FM systems. This is an important conceptual aspect, because it means that locally the direction of the exchange bias is determined by the direction of the FM domain magnetization. If we consider an inhomogeneous magnetization distribution, i.e., a magnetic domain pattern in the FM, the resulting exchange bias will show up as an average over the domain magnetization directions. Such a domain structure can be easily obtained by cooling the system in zero or a weak magnetic field. In a very simplified picture, we can assume the magnetization state of the FM to decay into domains with magnetization directions || or anti-\| to the applied field direction, thereby resulting in two locally varying exchange-bias directions. This situation can be used to control the amount of loop shift as the relative amount of one type of domains dominates over the other. As a consequence, the exchange bias shift can be tuned either by cooling in zero field (path I) or by the strength of the cooling field (path II). Technologically the magnetization reversal mechanisms for 


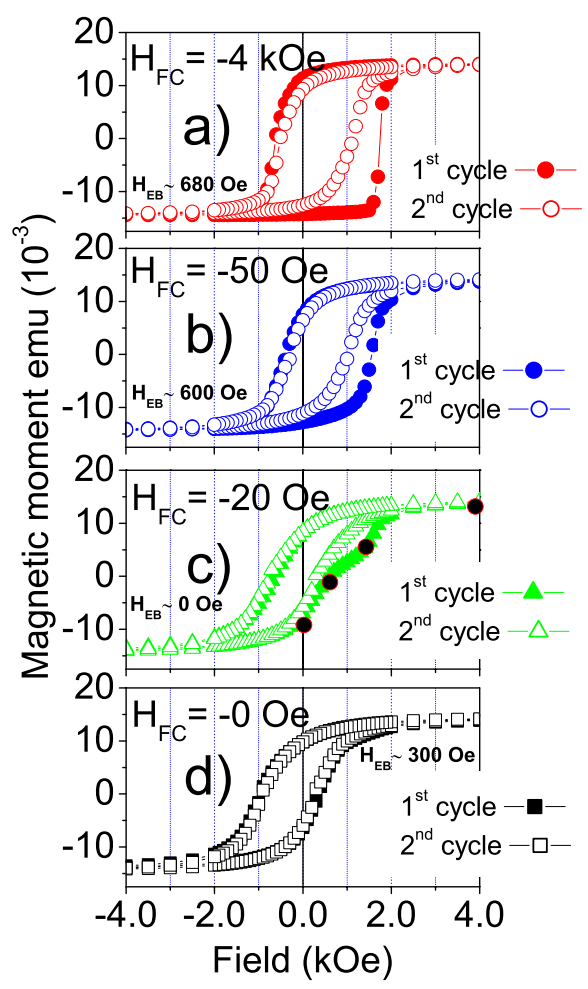

FIG. 1. (Color online) SQUID hysteresis loops of $[\mathrm{Co} / \mathrm{CoO} / \mathrm{Au}]_{16} \mathrm{ML}$ during the first (solid symbol) and second field cycles (open symbol) for $H_{\mathrm{FC}}$ of $-4.0 \mathrm{kOe},-50 \mathrm{Oe},-20 \mathrm{Oe}$, and 0 Oe. The closed black circles in panel (c) indicate the fields of neutron measurements shown.

the two states-which can have similar bias fields, but can be controlled by two different cooling processes-may be crucial, as both pathways I and II can be employed to achieve exchange biasing before and after the device fabrication.

So far we have only considered a symmetric reversal mechanism. Interestingly, also an "asymmetric" reversal mechanism has been observed in bilayers during the first field cycle, meaning that the loop shapes for the decreasing and increasing branch differ. This dependence on the magnetic history appears, for example, when domain nucleation and wall motion (nonuniform reversal) occur along the decreasing branch and magnetization rotation (uniform reversal) occurs along the subsequent increasing branch of the hysteresis loop. This difference between subsequent (partial) magnetization reversal loops is called the "training effect," an example of which is given in Fig. 1(a). The change in loop shape from the first to the second field cycle is obvious. Note that in this example the loop starts at negative saturation. Therefore, the increasing branch must then already be considered as trained, ${ }^{13}$ as it is the second half of the first cycle. On the other hand, during the second field cycle, the magnetization reversal is always symmetric and uniform. ${ }^{14}$ With respect to technological applications, controlling the training effect is important for the device performance.

There are only a few experimental techniques, which can address the magnetic aspects in both ferromagnets and antiferromagnets, and they employ either synchrotron radiation or neutrons. In particular, depth sensitive neutron scattering under grazing incidence with polarization analysis (PNR) has been proven decisive for the identification of reversal mechanisms in exchange bias systems. ${ }^{7,14-16}$ Due to the interaction between polarized neutrons and magnetic moments in the specimen, PNR is sensitive to the in-plane magnetization for a homogeneous film. We define the longitudinal magnetization component as parallel to the applied field direction $M \cos \phi_{\mathrm{A}}$ and the transversal magnetization component as perpendicular to the applied field direction $M \sin \phi_{\mathrm{A}}$, where $\phi_{\mathrm{A}}$ is the angle between the magnetization $M$ and the applied field $H_{a}$. Neutron scattering with polarization analysis can discriminate the longitudinal and transverse components of the magnetization. In the experiment four different cross sections can be distinguished, namely, the nonspin flip (NSF) $\left(R_{++}\right.$and $\left.R_{--}\right)$and spin flip (SF) channels $\left(R_{+-}\right.$and $\left.R_{-+}\right)$. Here + and - signs are used to distinguish the intensity contributions $R$ representing a polarization component \| or anti-\| to the guiding field, respectively. $R_{++/--}$contains the sum/difference between the nuclear and magnetic scattering, whereas the SF signal contains only the magnetic information. A magnetization rotation is identified by a significant increase in the specular SF reflectivities, which corresponds to the formation of in-plane magnetization components developing perpendicular to the guiding field $H_{a}$, which is applied collinear to $H_{\mathrm{FC}}$. On the other hand, magnetic reversal by domain nucleation and propagation does not provide enhanced SF intensities, because the local magnetization $M$ is always collinear to $H_{a}$.

In this paper, we show that the training effect can be strongly influenced by the field cooling procedure. For $H_{\mathrm{FC}}$ $\sim H_{C}$ the coercive field of the ferromagnet, one may achieve a special situation with a coexistence of two FM states: the first one is similar to the (multidomain) state determined by the FM magnetization at $T_{B}$ when the system is cooled without any field, whereas the second state is determined by the presence of a cooling field, i.e., a single domain state. PNR has been used to probe the magnetization reversal of buried moments at the interfaces in this particular situation, revealing different reversal mechanisms for the two different states of the FM.

\section{SAMPLES AND EXPERIMENTAL DETAILS}

We have investigated multilayers of the composition $\mathrm{SiO}_{2} /[\mathrm{Co}(9.0 \mathrm{~nm}) / \mathrm{CoO}(7.0 \mathrm{~nm}) / \mathrm{Au}(25.0 \mathrm{~nm})]_{16}$ for the untrained and trained state with different field-cooling options. The multilayer structure has been chosen in order to enhance the signal of the neutrons scattered off the FM/AF interface. The thick Au spacer layer ensured that we have a sequence of comparable interfaces throughout the thickness of the multilayer. This was also confirmed by $\mathrm{x}$-ray reflectivity measurements. The polycrystalline samples were prepared by dc-magnetron sputtering of $\mathrm{Co}$ and $\mathrm{Au}$, and by using our standard ultraviolet (UV) light-assisted oxidation procedure. ${ }^{7,8}$ Magnetization loops were measured by means of a superconducting quantum interference device (SQUID) at $10 \mathrm{~K}$ after field cooling in different external fields from its positive remanent state (after saturating in a positive field) at room temperature to $10 \mathrm{~K}$, i.e., well below the blocking tem- 
perature $T_{B} \approx 220 \mathrm{~K}$ of $\mathrm{CoO} .{ }^{7}$ The coercive field of the multilayers was determined as $H_{C} \approx 18$ Oe at room temperature (RT).

The PNR data have been measured at AMOR at the Swiss spallation neutron source (SINQ) of the Paul Scherrer Institute (PSI). ${ }^{19}$ All four polarization channels of the specular reflectivities were recorded by a single ${ }^{3} \mathrm{He}$ detector at different external fields $H_{a}$. The measurements were performed in time-of-flight (TOF) mode for two different incident angles $0.4^{\circ}$ and $1.0^{\circ}$ covering the normal wave vector transfer $Q_{\perp}=\frac{2 \pi}{\lambda}\left[\sin \left(\alpha_{i}\right)+\sin \left(\alpha_{f}\right)\right]$ range from 0.005 to $0.1 \AA^{-1}$. In specular geometry the angle of incidence $\alpha_{i}$ equals the final angle $\alpha_{f}$, and $\lambda$ is the wavelength from the spectrum selected by the chopper system. All measurements have been done after the sample was cooled to $10 \mathrm{~K}$ from room temperature by a closed-cycle refrigerator in the presence of defined cooling field provided by an electromagnet.

\section{RESULTS}

\section{A. Hysteresis loops}

Figures 1(a)-1(d) shows the SQUID hysteresis loops for different magnitudes of the cooling field. During the first cycle of the field sweep, at $H_{\mathrm{FC}}=-50$ Oe (blue curve), the FM magnetization is almost completely aligned along the FC direction (65\% of saturation magnetization at remanence as compared to $80 \%$ for $H_{\mathrm{FC}}=-4000 \mathrm{Oe}$ ). The pinning direction in the AF is determined by the exchange coupling. This results in a "negative shift" of the loop, which is typically observed in these samples. Furthermore, during the second cycle, we see a decrease in the magnitude of the exchange bias from the value that has been observed during the first cycle. This behavior is related to the training effect. ${ }^{13}$ Here also, the decreasing branch (first half of the first cycle) is untrained (nonuniform reversal) and increasing branch (second half of the first cycle) is trained (uniform reversal).

For $H_{\mathrm{FC}}=0$ Oe (black curve), a shift in the hysteresis loop (first cycle) is observed after cooling the system in the positive remanent state $\left(+m_{R}\right)$ of the FM. This shift is very similar to what has been reported earlier ${ }^{11}$ and is usually observed when samples are cooled in positive fields. One may note that as we always start our field sweep from negative saturation, the state of the system during its first field cycle must be considered already trained (in the present case the system is cooled from a positive remanent state). We can observe an untrained state only, if the cooling field points into the same direction as the starting field for the hysteresis cycle. This may explain, why we do not observe any further training during the subsequent second field cycle.

One may note that when the cooling field is more than the coercive field of the specimen at RT, as in cases shown in Figs. 1(a) and 1(b), then the cooling field is pointing into the same direction as the starting field (negative in this case) and we observe an untrained state during the first half of the first cycle. However, such is not the case when the cooling field is close to or below the coercive field Figs. 1(c) and 1(d). Here, we have to consider the remanent states $\left(+m_{R}\right.$ or $\left.-m_{R}\right)$ of the FM which become the deciding factor. Thus the decreasing

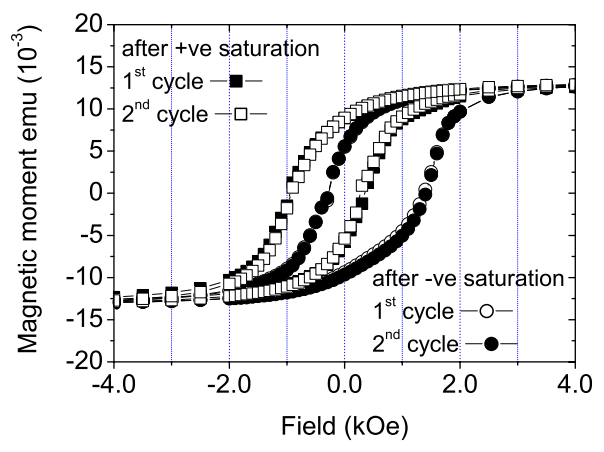

FIG. 2. (Color online) SQUID hysteresis loops of $[\mathrm{Co} / \mathrm{CoO} / \mathrm{Au}]_{16} \mathrm{ML}$ during the first (solid symbol) and second field cycles (open symbol) for $H_{\mathrm{FC}}=0 \mathrm{Oe}$. The loops correspond to a zero field cooling state after positive saturation and negative saturation at $\mathrm{RT}$.

branch (first half of the first cycle) shows a trained state (uniform reversal) behavior as we have cooled the system from $+m_{R}$.

However, even when we cool the system in the $-m_{R}$ state of the FM magnetization, we do not observe any net training. SQUID hysteresis loops of the multilayer during the first and second field cycles for $H_{\mathrm{FC}}=0$ Oe are shown in Fig. 2. The loops correspond to the zero-field cooling state after positive saturation $\left(+m_{R}\right)$ and negative saturation $\left(-m_{R}\right)$ at room temperature, respectively. The loops for $+m_{R}$ are already trained (as explained earlier), while the loops for $-m_{R}$-though untrained during the first cycle (first half of the first cycle) — do not show training at all. The observation of a macroscopic training from a comparison of the hysteresis loops is a usual feature in exchange bias system. ${ }^{17}$ Therefore, this suppression of training for zero-field cooling is surprising and interesting. It indicates that the state of the interface is virtually unaffected by the field cycling, as long as the sample has not been subjected to a cooling field. This suppressed training for zero-field cooling can therefore be used for controlling exchange bias systems even after the device fabrication and this can be applicable for any number of field cycles.

A particularly interesting behavior appears for a configuration, where the system had been cooled from $+m_{R}$ with $H_{\mathrm{FC}}=-20$ Oe (green curve), i.e., close to the coercive field $H_{C}$ of the layers. From the shape of the hysteresis loop (first cycle) one can derive that the magnetization reversal must involve two different pathways: in the upper part of the loop the curve is shifted opposite to the cooling field orientation (identified by the usual negative shift), while in the lower part the curve is basically unaffected by the increase in $H_{\mathrm{FC}}$. This could be due, for example, to two different types of domain configurations, i.e., in the extreme case a singledomain vs a multidomain state. When the cooling field is increased from zero to $\left(H_{\mathrm{FC}} \simeq H_{C}\right)$, an equal population of two micromagnetic configurations is to be expected. Therefore, the negative shift of the upper component can be attributed to the usual pinning of the uncompensated AF spins to the exchange field of the FM, which is aligned by the strength of the cooling field. ${ }^{18}$ The lower component, on the other hand, still retains the zero-field cooling state, as the AF 


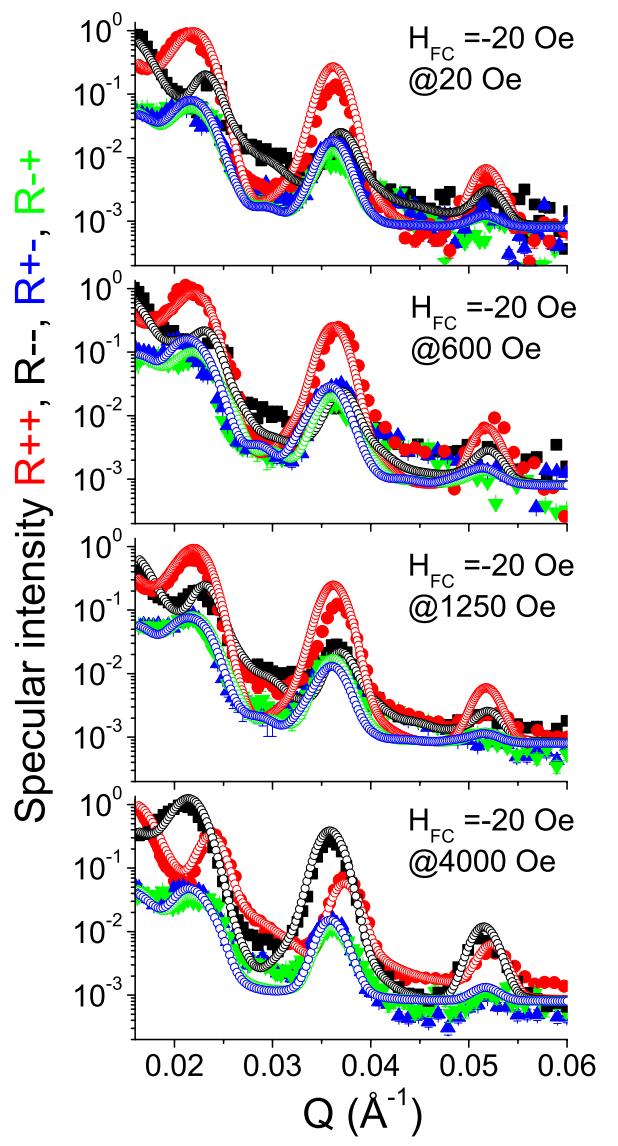

FIG. 3. (Color online) Specular reflectivity patterns NSF: $\left[R_{++}\right.$ (red/black circle) and $R_{--}$(black square) $]$and SF: $\left[R_{-+}\right.$(green/light gray triangle) and $R_{+-}$(blue/black down triangle)] along with their fits (open circle) for $[\mathrm{Co} / \mathrm{CoO} / \mathrm{Au}]_{16} \mathrm{ML}$ for $H_{\mathrm{FC}}=-20 \mathrm{Oe}$.

spins are coupled to the initial magnetization state of the FM at $T_{B}$. This is even more clear as we follow the second field cycle. Here, the upper component is seen to be trained, while the lower component is virtually unaffected by the field cycling (just as it was seen for the zero-field cooling case when the sample was already trained during the first field cycling). Therefore, we can differentiate between the two types of interface exchange coupling and exchange biase contributions forming in our experiments: one that is determined by the strength of the cooling field $H_{\mathrm{FC}}\left(\mathcal{D}_{\mathrm{FC}}\right)$ and a second one $\left(\mathcal{D}_{0}\right)$, which is established without a cooling field at all.

With the increase in cooling field strength, $H_{\mathrm{FC}}$ $>-20$ Oe, the minimum energy configuration realized for zero-field cooling is altered, leading to the gradual disappearance of the contribution $\mathcal{D}_{0}$ tuned without the cooling field. Therefore, the loop shape (during the first cycle) for $H_{\mathrm{FC}}=$ -20 Oe is particularly interesting. It represents a state where one can observe partial pinning of the AF spins to the FM magnetization, which is determined by the state of FM magnetization above the blocking temperature and which is influenced by a cooling field. We investigate the magnetization reversal of this buried interfacial moments by depth-sensitive PNR. The absence of any sudden jump in the SQUID signal (measured in steps of $100 \mathrm{Oe}$ ) in the decreasing branch below $H_{\mathrm{FC}}=-20$ Oe apparently indicates that the magnetization

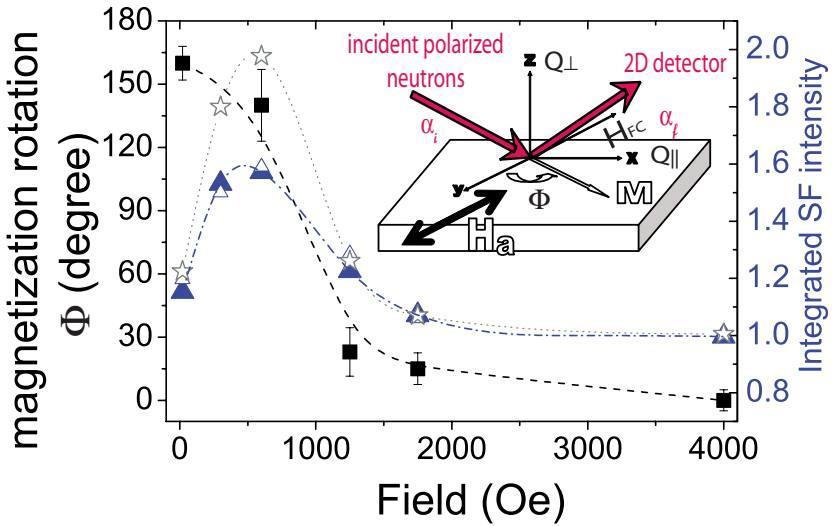

FIG. 4. (Color online) Integrated SF intensity for varying $H_{a}$ measured (closed triangle) from the sample and from the simulated patterns corresponding to $\approx 25 \%$ (open triangle) and $100 \%$ (open star) of the net magnetization are shown for comparison along the decreasing branch of the hysteresis loop normalized to that in saturation. The angular variation of the magnetization (closed square) as deduced from the fits to the patterns are also shown alongside. The lines are guiding the eye.

$M_{\mathrm{FM}}$ reverses via a uniform mechanism, which proceeds further via a nonuniform mechanism only around some critical field in the range of $H_{\mathrm{FC}}=-20$ to $-50 \mathrm{Oe}$ (measured with similar field steps).

\section{B. Neutron scattering measurements}

The specular reflectivity patterns are shown in Fig. 3 for $H_{\mathrm{FC}}=-20 \mathrm{Oe}$ [taken at four different $H_{a}$ values along the decreasing branch of the hysteresis loop indicated by closed black circles in Fig. 1(c)] together with their least-square fits. ${ }^{715}$ The three peaks of the ML in the NSF channels are the first, second, and third order Bragg reflections of the ML. $R_{++}$dominates over $R_{--}$for all fields before the reversal. This is related to a net magnetization, which is collinear to $H_{a}$ during the first field cycle.

Figure 4 shows the integrated SF intensity for various fields along the decreasing branch of the hysteresis loop for neutron measurements during the first field cycle. The angular variation $(\Phi)$ of the net magnetization, as deduced from the fits to the specular reflectivity patterns, is also shown alongside. At $4.0 \mathrm{kOe}$, which is the saturation field, the SF signal can be solely attributed to the $\approx 5 \%$ polarization inefficiencies of the setup.

We now compare the SF signals of the measured reflectivity patterns at all other fields with that measured at 4.0 kOe. Here, we observe an increase in the SF signal by $\approx 60 \%$ compared to the values at magnetic saturation. This increase appears over a field range $(0-1.0 \mathrm{kOe})$, where one expects a reversal of that proportion $\mathcal{D}_{0}$, which has been determined by the remanent magnetization above $T_{B}$, i.e., without the influence of any cooling field. We find that around $\approx 25 \%$ of the net magnetization is rotating and an intensity maximum is observed around 600 Oe.

For a higher field range (above $1.0 \mathrm{kOe}$ of $H_{a}$ ), no significant SF signal is observed. This is indicative of the fact that 
a nonuniform reversal, i.e., via domain wall and nucleation process takes place in this field range. Interestingly, in this field regime, one expects the effect to be related to the reversal of that proportion $\mathcal{D}_{\mathrm{FC}}$ of the magnetization that has been determined by the cooling field strength [indicated by the partial loop shift in Fig. 1(c)]. However, for the trained state, a uniform reversal is observed for all field ranges (not shown). Thus, we find two different mechanism of magnetization reversal depending upon the field cooling strength or the domain state of the FM above $T_{B}$.

\section{DISCUSSION}

One of the key issues that has emerged in the discussion of exchange biasing is the role of disorder and frustration in ferromagnet/antiferromagnet combinations. Several models have been considered (starting from the unsatisfactory macroscopic models proposed by Meiklejohn-Bean ${ }^{1}$ and Mauri ${ }^{20}$ ) to account for the shift and the magnitude of the exchange bias.

(a) In the approach of Malozemoff, ${ }^{21}$ within a mesoscopic model (nm scale: compositional correlation length is smaller than the exchange length), exchange bias is attributed to the formation of domain walls in the AFM perpendicular to the FM/AFM interface due to interface roughness. However, the formation of domains in the AFM only due to interface roughness is unlikely to occur, because the creation of the domain walls is energetically unfavorable.

(b) By calculating the AF-FM spin structure near the interface, within the microscopic model (atomic scale), Koon ${ }^{22}$ considered a spin-flop coupling between a FM and the compensated AFM (perpendicular orientation) as responsible for exchange bias. However, Schulthess and Butler ${ }^{23}$ showed that spin-flop coupling alone cannot account for this effect as exchange bias is only obtained if uncompensated AFM spins are assumed at the interface.

(c) In another approach, the AF layer is assumed to be composed of magnetically uncoupled crystallites by Takano and Berkowitz. ${ }^{24}$ A refinement of this model has been proposed by Stiles and $\mathrm{McMichael}^{25}$ by considering rotatable (responsible for coercivity increase) and nonrotatable grains (responsible for unidirectional anisotropy).

(d) Recently it has been shown (on the basis of a classical Heisenberg or rigid Ising model) that the observed exchange bias is primarily not due to disorder or defects at the interface. Instead, the full antiferromagnetic layer must be involved as the domain-state develops due to the dilution of the AFM. ${ }^{26}$ The domains are pinned at the impurity sites thereby reducing considerably the energy necessary to create a wall.

(e) The model of Lee and Okabe ${ }^{27}$ combined the granular model of Stiles and McMichael with the domain-state model. They treated the case of explicit domains in the AF considering a free boundary on the surface of each grain and an effective interaction field thereby computing the mean field magnetization. In their calculation the hysteresis loop remain unshifted for zero-field cooling when cooled from a demagnetized state.

Our experimental results can be reasonably well explained within the framework of the domain state model and

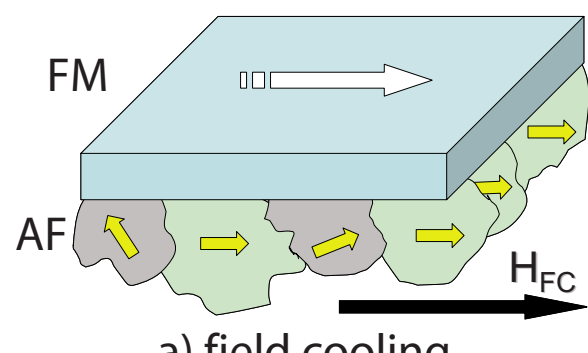

a) field cooling

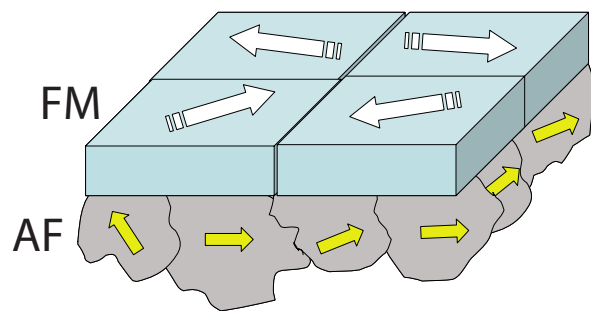

b) zero field cooling

FIG. 5. (Color online) Schematic of the AF-FM layer magnetizations for (a) field cooling and (b) zero field cooling situations. The arrows indicate the magnetization directions of the FM and AF layer.

its expansion by Lee and Okabe. In the domain state model, the exchange field is provided by the remanent magnetization of the domains in the AF during different field cooling procedures. This remanent magnetization is influenced by the magnetization state of the FM (i.e., if the FM is divided into almost equal proportion of domains or it is already in a saturated state) on top of the AF layer as they are coupled to each other by the exchange interaction. During magnetization reversal of the FM layer a domain wall can wind up in the AF layer, the energy of which depends upon the uniaxial anisotropy within the AF layer. A schematic picture of the AF-FM layer magnetizations for (a) zero field cooling and (b) field cooling situations is shown in Fig. 5.

Very recently an explanation of training effect has been proposed by Brems et al. ${ }^{29}$ They have shown that the deviation of the average interfacial magnetization vector of the $\mathrm{AF}$ CoO-grains from their initial $H_{\mathrm{FC}}$ direction, can create a sufficiently large torque leading to rotation of the FM magnetization. The extent of such a deviation is usually large enough after the second field cycling, as not all magnetization vectors rotate back to their initial directions. We can consider in our case, that the torque acting on the system is due to an effective field and the applied field causing the magnetization reversal. The effective field can be expressed as the vector sum of the unidirectional anisotropy and the rotatable anisotropy (rotation of hysteretic grains). ${ }^{28}$

When the system is cooled down in a saturating field (FM in a single-domain state $\mathcal{D}_{\mathrm{FC}}$ ), the unidirectional anisotropy is the dominating entity. This is because the interfacial magnetization vector of almost all AF grains (depending upon the strength of the cooling field) are closely aligned to the $H_{\mathrm{FC}}$ direction [Fig. 5(a)]. This alignment is obviously disturbed after a complete field sweep as the interfacial magnetization vector of a considerable portion of $\mathrm{AF}$ grains is now randomly distributed. 


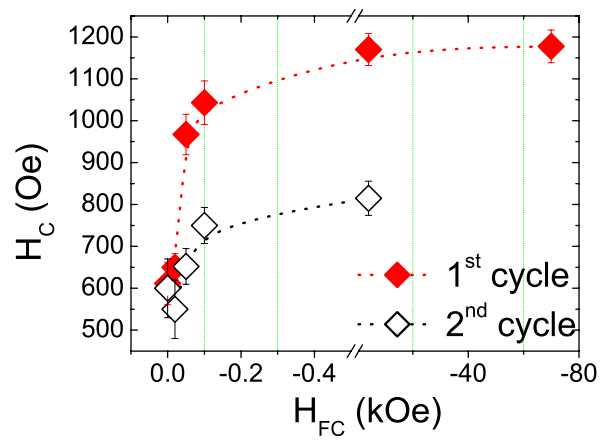

FIG. 6. (Color online) $H_{\mathrm{FC}}$ variation with $H_{a}$ during the first and second field cycles. The lines are guiding the eye.

When the system is cooled in zero field (FM in a multidomain state $\mathcal{D}_{0}$ ), the interfacial magnetization vector of each AF grain is randomly distributed [Fig. 5(b)]. The average direction, however, lies along the saturating field direction (the remanent magnetization at RT is $\approx 35 \%$ lower than that at saturation). Here, the rotatable anisotropy dominates over the unidirectional anisotropy. During the second field cycle, the interfacial magnetization vector of almost all AF grains remain similar to the state during the first field cycle. In Fig. 6 we show the variation $H_{c}$ (half width of the hysteresis loop) with $H_{a} . H_{c}$ is seen to be increasing with $H_{a}$ below $1.0 \mathrm{kOe}$ and saturates above. This increase indicates a rotation of the grains (excess magnetization within the grains) ${ }^{25}$ There is no significant difference in the $H_{c}$ for the first and second cycles below $H_{a}=20$ Oe indicating similar population of rotating grains. Thus we observe a suppression of training.

\section{CONCLUSION}

In conclusion, we have shown that the extent of training in exchange bias can be strongly determined by a balance of different micromagnetic configurations. These magnetic configurations may be realized by (i) cooling in a remanent state of the FM magnetization at $T_{B}$ results in a multidomain state, whereas (ii) cooling in a saturating field $H_{\mathrm{FC}}$ results in a single domain state. For zero-field cooling, we observe no macroscopic evidence of training, whereas for a cooling field $>H_{C}$, usual training was observed. For a critical cooling field $\left(H_{\mathrm{FC}} \sim H_{C}\right)$, we find a coexistence of the two magnetic configurations, resulting in a peculiar behavior of the exchange bias. To our knowledge, this is the first experimental observation where training is strongly influenced by the field cooling procedure. Using PNR, we could quantitatively differentiate between nonuniform magnetization reversal (when the FM magnetization is in a single-domain state) from that of uniform reversal of the net magnetization (when the FM magnetization is in a multidomain state) buried at such FM-AF interfaces. We could explain our observations within the framework of the domain state model combined with a consideration of antiferromagnetic grains in polycrystalline systems. $^{25}$
*Author to whom correspondence should be addressed. A.Paul@fzjuelich.de

${ }^{1}$ W. H. Meiklejohn and C. P. Bean, Phys. Rev. 102, 1413 (1956).

${ }^{2}$ I. N. Krivorotov, C. Leighton, J. Nogués, Ivan K. Schuller, and E. D. Dahlberg, Phys. Rev. B 65, 100402(R) (2002).

${ }^{3}$ A. Paul, E. Kentzinger, U. Rücker, and Th. Brückel, Phys. Rev. B 74, 054424 (2006).

${ }^{4}$ M. Kiwi, J. Magn. Magn. Mater. 234, 584 (2001).

${ }^{5}$ H. Ohldag, H. Shi, E. Arenholz, J. Stöhr, and D. Lederman, Phys. Rev. Lett. 96, 027203 (2006).

${ }^{6}$ G. Scholten, K. D. Usadel, and U. Nowak, Phys. Rev. B 71, 064413 (2005).

${ }^{7}$ A. Paul, E. Kentzinger, U. Rücker, and Th. Brückel, Phys. Rev. B 73, 092410 (2006).

${ }^{8}$ A. Paul, D. Bürgler, M. Luysberg, and P. Grünberg, Europhys. Lett. 68, 233 (2004).

${ }^{9}$ M. Gierlings, M. J. Prandolini, H. Fritzsche, M. Gruyters, and D. Riegel, Phys. Rev. B 65, 092407 (2002).

${ }^{10}$ F. Radu, M. Etzkorn, R. Siebrecht, T. Schmitte, K. Westerholt, and H. Zabel, Phys. Rev. B 67, 134409 (2003).

${ }^{11}$ P. Miltényi, M. Gierlings, M. Bamming, G. Günthererodt, J. Nogués, M. Gruyters, C. Leighton, and I. K. Schuller, Appl. Phys. Lett. 75, 2304 (1999).

${ }^{12}$ H. Ouyang, K.-W. Lin, C.-C. Liu, Shen-Chuan Lo, Y.-M Tzeng, J.-Y. Guo, and J. van Lierop, Phys. Rev. Lett. 98, 097204 (2007).
${ }^{13}$ J. Nogués and I. K. Schuller, J. Magn. Magn. Mater. 192, 203 (1999).

${ }^{14}$ W.-T. Lee, S. G. E. te Velthuis, G. P. Felcher, F. Klose, T. Gredig, and E. D. Dahlberg, Phys. Rev. B 65, 224417 (2002).

${ }^{15}$ A. Paul, E. Kentzinger, U. Rücker, D. E. Bürgler, and P. Grünberg, Phys. Rev. B 70, 224410 (2004); Eur. Phys. J. B 45, 249 (2005).

${ }^{16}$ M. R. Fitzsimmons, P. Yashar, C. Leighton, I. K. Schuller, J. Nogues, C. F. Majkrzak, and J. A. Dura, Phys. Rev. Lett. 84, 3986 (2000).

${ }^{17}$ U. Nowak and K. D. Usadel, J. Keller, P. Miltènyi, B. Beschoten, and G. Güntherodt, Phys. Rev. B 66, 0144302002.

${ }^{18}$ J. Camarero Y. Pennec, J. Vogel, S. Pizzini, M. Cartier, F. Fettar, F. Ernult, A. Tagliaferri, N. B. Brookes, and B. Dieny, Phys. Rev. B 67, 020413(R) (2003).

${ }^{19} \mathrm{http} / / /$ kur.web.psi.ch/amor/

${ }^{20}$ D. Mauri, H. C. Siegmann, P. S. Bagus, and E. Kay, J. Appl. Phys. 62, 3047 (1987).

${ }^{21}$ A. P. Malozemoff, Phys. Rev. B 35, 3679 (1987).

${ }^{22}$ N. C. Koon, Phys. Rev. Lett. 78, 4865 (1998).

${ }^{23}$ T. C. Schulthess and W. H. Butler, Phys. Rev. Lett. 81, 4516 (1998).

${ }^{24}$ K. Takano, R. H. Kodama, A. E. Berkowitz, W. Cao, and G. Thomas, Phys. Rev. Lett. 79, 1130 (1997).

${ }^{25}$ M. D. Stiles and R. D. McMichael, Phys. Rev. B 59, 3722 (1999). 
${ }^{26}$ P. Miltènyi, M. Gierlings, J. Keller, B. Beschoten, G. Güntherodt, U. Nowak, and K. D. Usadel, Phys. Rev. Lett. 84, 4224 (2000); U. Nowak, A. Misra, and K. D. Usadel, J. Magn. Magn. Mater. 240, 243 (2002).

${ }^{27}$ H. K. Lee and Y. Okabe, Phys. Rev. B 73, 140403(R) (2006).
${ }^{28}$ T. Gredig, I. N. Krivotov, C. Merton, A. M. Goldman, and E. D. Dahlberg, J. Appl. Phys. 87, 6418 (2000).

${ }^{29}$ S. Brems, K. Temst, and C. Van Haesendonck, Phys. Rev. Lett. 99, 067201 (2007). 